\title{
GLOBAL MAGNESIUM RESEARCH: STATE-OF-THE-ART AND WHAT'S NEXT?
}

\author{
Karl Ulrich Kainer \\ Helmholtz Zentrum Geesthacht, Magnesium Innovation Center; Max-Planck-Straße 1, 21502 Geesthacht, Germany \\ Keywords: magnesium research and development, global situation, issues and challenges
}

\begin{abstract}
In recent years magnesium and its alloys have been successfully introduced into weight-saving applications in the transportation industries in order to reduce fuel consumption and greenhouse gas emissions as well as to increase the performance of modern cars. Besides advantages, e.g. superior specific strength and excellent processability, applications of magnesium alloys are limited due to their inferior properties at elevated temperatures, e.g. low creep resistance and reduced corrosion behavior, especially when in galvanic contact with other metallic materials.
\end{abstract}

Current developments are revealing possibilities to improve these properties by using modern alloys and processing routes. While the majority of industrial applications utilize cast products, the use of wrought magnesium alloys is still at an early stage. Within the framework of ongoing research and development, the corrosion behavior of both cast and wrought magnesium materials in standalone uses or in galvanic couples with other metallic materials is gaining increasing attention. New coating systems tailored to selected applications will have to be developed in order to increase the usage of magnesium alloys in the transportation industries in the future. This work also needs to be coordinated with new processes for joining magnesium alloys with similar and dissimilar metals and alloys, to achieve a broad spectrum of materials that fulfill the requirements given by the applications.

After years of intensive research in Europe, North America, Australia and Asia, an increase in these activities has taken place in recent years, in particular in China and Korea. The magnesium industry has to face new challenges with regard to market issues, the breakdown of the Western magnesium industry and finally the carbon footprint discussion of the life-cycle assessment of components for the transportation industry.

This presentation will first address these issues and challenges, then discuss new developments and finally show some examples of new applications. In the conclusions, gaps and challenges will be analyzed and recommendations for sustainable research and development will be given. 